\title{
Cardioprotective effects of HDL cholesterol called into question
}

The results of a Mendelian randomization analysis published in the Lancet indicate that HDL cholesterol might not have a causal role in preventing coronary heart disease (CAD), as has been hypothesized. "These results suggest that ... just because an intervention raises HDL cholesterol, we cannot automatically assume that risk for heart attack will be lowered," says investigator Dr Sekar Kathiresan.

Often referred to as 'good' cholesterol, high levels of HDL cholesterol have been associated with reduced cardiovascular risk in observational studies. The proposed cardioprotective mechanisms of HDL include its role in reverse cholesterol transport and in modulating inflammation. However, unlike LDL cholesterol, a causative link between HDL cholesterol and cardiovascular risk has not been established.

Voight and colleagues analyzed the association between single nucleotide polymorphisms (SNPs) known to be associated with increased HDL-cholesterol levels and risk of CHD. The researchers applied the same analysis to LDL cholesterol to validate their methodology. All patient data was from individuals of European or South Asian descent. Two indicators of genetically raised HDL-cholesterol level were tested in the Mendelian analysis. First, a previously identified SNP that induces loss of function in the gene encoding endothelial lipase (LIPG Asn396Ser). Secondly, a genetic risk score composed of 14 SNPs. Importantly, the SNPs used in both these analyses only effect the level of HDL cholesterol, and do not have an impact on other lipids or known cardiovascular risk factors. This approach minimizes the chance that the results might be confounded by pleiotropic effects.
In four prospective cohort studies, carriers of the LIPG Asn396Ser allele had significant increases in HDL-cholesterol level (ranging from $0.08 \mathrm{mmol} / 1$ to $0.28 \mathrm{mmol} / \mathrm{l}$ ). However, among 50,763 participants of six prospective studies, LIPG Asn396Ser carrier status was not associated with a reduced risk of myocardial infarction. Neither did this SNP confer a cardioproective effect among 65,557 individuals enrolled in 14 case-control studies. Similarly, a 1 SD increase in HDL-cholesterol level with the genetic risk score was not associated with a reduced risk of myocardial infarction in 53,146 cases and controls in the CARDIoGRAM study.

By contrast, however, SNPs that conferred genetically increased levels of LDL cholesterol were shown to be causally related to increased risk of myocardial infarction. Thus, the analysis seems to have had the power to demonstrate an association where one exists.

Voight et al. note that one common genetic variation at the cholesterol ester transfer protein (CETP) gene did reduce the risk of myocardial infarction by approximately $4 \%$. However, as this variant raises HDL-cholesterol level and lowers LDL-cholesterol level, the individual effects cannot be distinguished from each other.

"The key question now is, are there any genes that affect both HDL and risk for myocardial infarction?" Concludes Dr Kathiresan. "If we identify such a gene, it might be useful as a therapeutic target."

\section{Alexandra King}

Original article Voight, B. F. et al. Plasma HDL cholesterol and risk of myocardial infarction: a Mendelian randomisation study. Lancet doi:10.1016/ S0140-6736(12)60312-2 